\title{
Differential Effects of Antioxidants on the In Vitro Invasion, Growth and Lung Metastasis of Murine Colon Cancer Cells
}

\author{
Masaru Ogasawara, * Takayuki Matsunaga, and Hideyo Suzuki \\ Toyama Prefectural Institute for Pharmaceutical Research; 17-1 Nakataikouyama, Imizu, Toyama 939-0363, Japan. \\ Received August 23, 2006; accepted October 25, 2006; published online October 27, 2006
}

\begin{abstract}
We conducted a comparative study of 20 antioxidants including antioxidative vitamins and polyphenols to examine their inhibitory activities against the in vitro invasion, growth and experimental lung metastasis of murine colon 26-L5 carcinoma cells. Among the compounds tested, epigallocatechin gallate (EGCG), gallocatechin gallate and genistein exhibited significant reductions at $77 \%, 46 \%$ and $44 \%$ in tumor metastasis by an intraperitoneal administration for $5 \mathrm{~d}$ beginning at $3 \mathrm{~d}$ before tumor inoculation, respectively. Quercetin also showed a slight but not statistically significant inhibition. $\alpha$-Tocopherol, $\beta$-carotene, ascorbic acid and 2 EGCGrelated compounds of epicatechin gallate and epigallocatechin had no effect. EGCG also inhibited tumor metastasis dose-dependently with $98 \%$ suppression at $2 \mu \mathrm{mol}$; and an almost equivalent inhibition was also produced by only pre-administration of EGCG at the same dose before tumor inoculation. EGCG significantly inhibited tumor cell invasion and proliferation, but its inhibition of these activities was much less effective than that of other compounds which did not show any antimetastatic effect. No statistically significant relationship was observed between the radical scavenging activities of the test compounds and their rates of inhibition of tumor metastasis. The antimetastatic mechanism of EGCG thus seems to be independent of its inhibition of tumor invasion and growth, as well as its radical scavenging activity. Our results suggest that EGCG is potentially beneficial for tumor metastasis inhibition.
\end{abstract}

Key words epigallocatechin gallate; antioxidant; tumor metastasis

Cancer metastasis is a major cause of death in cancer patients, and its blockade is believed to enable cancer patients to live longer. ${ }^{1)}$ Thus, it is important to find promising antimetastatic agents.

A variety of compounds have been shown to be benefitial for the inhibition of cancer metastasis. Some of them involve mechanisms based on the invasive inhibition of tumor cells by interfering with tumor adhesion to extracellular matrix components, ${ }^{2)}$ inhibiting tumor cell-associated protease activity, ${ }^{3)}$ or reducing the motile activity of tumor cells. ${ }^{4)}$ Other compounds work through immunostimulation ${ }^{5)}$ or through the inhibition of tumor-endothelial or -platelet interaction. ${ }^{6,7)}$ Although these antimetastatic compounds have great potential for clinical applications, it remains critical to find compounds which may be much more effective.

Antioxidants includes a variety of compounds having the ability of capturing reactive oxygen species; which are such as antioxidative vitamins and polyphenols. Antioxidants therefore have been proposed to have potential benefits for the prevention and treatment of diseases associated with reactive oxygen species. Especially in the cancer research field, antioxidants have been demonstrated to be effective in reducing the risk of carcinogenesis partly based on their antioxdative activity. ${ }^{8}$ Some antioxidants have also been shown to have other anti-cancer properties, which are inhibitory activities on cancer cell-derived proteases, cancer cell invasion and growth, and angiogenesis. ${ }^{9}$ Inhibitory activities on cancer metastasis have also been reported for some antioxidative vitamins, ${ }^{10,11)}$ their analogues ${ }^{12,13)}$ and some polyphenols ${ }^{14-19)}$ in animal models; and their inhibitory mechanisms have been suggested to involve the inhibition of cancer cell invasion or angiogenesis, or the induction of apoptosis of cancer cells.

It is important to note here that most of the in vivo experiments showing the efficacy of the compounds have been conducted in an experimental design evaluating their effect on the development of tumor micrometastasis, which suggests therapeutic values of compounds. In contrast, preventive activity of those compounds on the formation of tumor metastasis has not fully understood.

The present comparative study examined the inhibitory activities against the in vitro invasion, growth and experimental lung metastasis of murine colon carcinoma cells of 20 antioxidants, including antioxidative vitamins and polyphenols.

\section{MATERIALS AND METHODS}

Materials Antioxidants and Lentinan were purchased from Wako Pure Chemical Industries, Ltd. (Osaka, Japan) and Yamanouchi Pharmaceutical Co., Ltd. (Tokyo, Japan), respectively. Antioxidants were dissolved in dimethyl sulfoxide (DMSO) at a concentration of $100 \mathrm{~mm}$ as a stock solution. In each in vitro experiment, antioxidants were diluted with DMSO and added into medium to be the final concentration of $1 \%$ DMSO. In each in vivo experiment, antioxidants were used after dilution with DMSO to be the appropriate doses. Lentinan was dissolved in $\mathrm{Ca}^{2+}$ and $\mathrm{Mg}^{2+}$-free phosphate-buffered saline.

Cells and Cell Culture Murine colon 26-L5 adenocarcinoma cell line, derived from colon 26 , was kindly provided by Professor I. Saiki (Toyama University, Institute of Natural Medicine, Toyama, Japan). The cell line is highly liver metastatic compared with the colon 26 parental cell line and has also been shown to metastasize to lungs potently in an experimental metastasis model. ${ }^{20}$ ) The cell line was maintained in RPMI-1640 (Gibco, Grand Island, NY, U.S.A.) supplemented with $10 \%$ fetal bovine serum (FBS), 2-mercaptoethanol, $100 \mathrm{U} / \mathrm{ml}$ penicillin and $0.1 \mathrm{mg} / \mathrm{ml}$ streptomycin.

Animals Inbred 6-week-old female BALB/c mice were purchased from Shizuoka Laboratory Animal Center (Hamamatsu, Japan). All mice were housed in a controlled environ- 
ment with a 12 -h light/dark cycle, a temperature of $24 \pm 2{ }^{\circ} \mathrm{C}$ and a humidity level of $55 \pm 10 \%$; they were given commercial food and tap water ad libitum. The study period began after an acclimatization period of 1 week. All animal experiments were carried out in accordance with the animal care policy of Toyama Prefectural Institute for Pharmaceutical Research.

Experimental Lung Metastasis Assay Colon 26-L5 cells $\left(4 \times 10^{4}\right)$ were injected intravenously into 5 mice per group. Twenty microliters per mouse of prepared test compounds were administered intraperitoneally 5 consecutive times beginning at $3 \mathrm{~d}$ before tumor inoculation to examine their effect on tumor metastasis formation. The control was vehicle administered in the same way. In some experiments, twenty mice were randomized into four groups: the first group was daily administered EGCG $(2 \mu \mathrm{mol} / 20 \mu \mathrm{l} / \mathrm{mouse})$ from $3 \mathrm{~d}$ before tumor inoculation to the next day of it; the second group was administered firstly EGCG $(2 \mu \mathrm{mol} / 20$ $\mu \mathrm{l} /$ mouse) for $3 \mathrm{~d}$ before tumor inoculation and subsequently vehicle for $2 \mathrm{~d}$ after the inoculation; the third group was administered firstly vehicle for $3 \mathrm{~d}$ before tumor inoculation and subsequently EGCG ( $2 \mu \mathrm{mol} / 20 \mu \mathrm{l} /$ mouse) for $2 \mathrm{~d}$ after the inoculation; and the fourth group was administered vehicle for $5 \mathrm{~d}$ as the control. Thirteen days after tumor inoculation, tumor nodules in the lungs were counted under a dissection microscope.

Cell Invasion Assay Tumor cell invasion through reconstituted basement membrane (Matrigel) was assayed following the methods previously reported by Saito et al. ${ }^{21)}$ Colon 26-L5 cells $\left(2 \times 10^{5}\right)$ suspended in RPMI-1640 medium containing $0.1 \%$ bovine serum albumin were pretreated with various concentrations of the test compounds for $30 \mathrm{~min}$ on ice, and then added to the upper compartment. Test compounds were also added to the lower compartment. The control was vehicle administered in the same way. After incubation for 5 $\mathrm{h}$ at $37^{\circ} \mathrm{C}$, the cells were fixed and stained with crystal violet. Cells that had invaded the lower surfaces of the filters were extracted, and the absorbance of the cell lysate was measured at $590 \mathrm{~nm}$.

Cell Proliferation Assay Colon 26-L5 cells $\left(5 \times 10^{3}\right)$ suspended in RPMI-1640 medium containing 5\% FBS were seeded into the wells of 96-multiwell plates with vehicle or various concentrations of the test compounds, and incubated at $37^{\circ} \mathrm{C}$ for $48 \mathrm{~h}$. The culture medium was then exchanged for fresh medium containing 10\% FBS and 10\% WST-1 solution (Wako), and the cells were incubated at $37^{\circ} \mathrm{C}$ for an additional $2 \mathrm{~h}$. The absorbance of each well was measured at $450 \mathrm{~nm}$.

Free Radical Scavenging Assay A 1,1-diphenyl-2picrylhydrazyl (DPPH) assay was performed following the methods previously reported by Blois. ${ }^{22}$ Briefly, the stable radical DPPH in ethanol was added to $2 \mathrm{ml}$ of ethanol containing different concentrations of the test compounds. Each mixture was incubated for $30 \mathrm{~min}$ at $37^{\circ} \mathrm{C}$. The absorbance of DPPH at $517 \mathrm{~nm}$ was then measured. Ethanol was used as a blank solution and DPPH solution in ethanol served as the control. The concentrations needed to reduce the initial amount of DPPH radical to half $\left(\mathrm{ED}_{50} \mathrm{~s}\right)$ were determined.

Statistical Analysis All data are expressed as the mean \pm S.D. Statistical differences were evaluated by twotailed $t$-test, and $p<0.05$ was considered to be significant.

\section{RESULTS}

Effect of Antioxidants on Tumor Lung Metastasis Twenty antioxidants were examined for their activity against lung metastasis of colon 26-L5 cells in mice at a dose of 1 $\mu$ mol (Fig. 1). Epigallocatechin gallate (EGCG), gallocatechin gallate and genistein were found to bring about significant reductions in tumor nodules in the lungs at $77 \%, 46 \%$ and $44 \%$, respectively. Quercetin showed a slight but not statistically significant inhibition. $\alpha$-Tocopherol, $\beta$-carotene and ascorbic acid had no inhibitory effect.

We further examined the dose-responsive effects of EGCG using the same animal model (Fig. 2A). EGCG was found to inhibit lung metastasis in a dose-dependent manner and

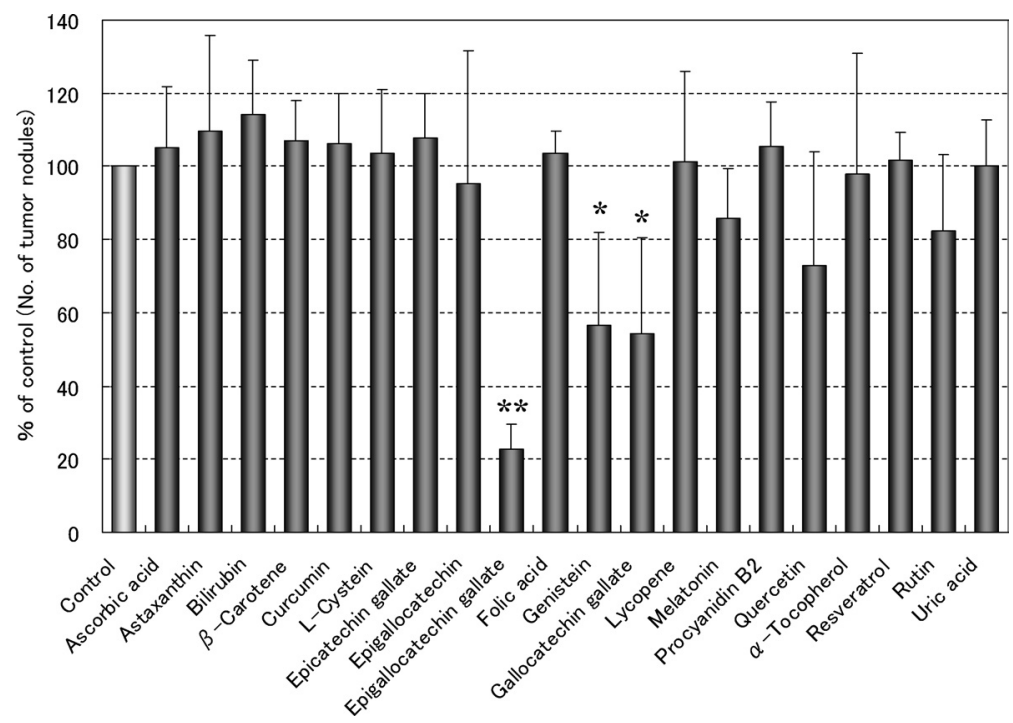

Fig. 1. Effects of Antioxidants on Lung Metastasis of Colon Carcinoma Cells in Mice

Colon 26-L5 cells were inoculated into mice through a tail vein. The test compounds were administered intraperitoneally into mice for $5 \mathrm{~d}$ beginning at $3 \mathrm{~d}$ before tumor inoculation. Thirteen days after tumor inoculation, tumor nodules on the surfaces of the lungs were counted. The data are expressed as the mean \pm S.D. of 5 mice. $* p<0.05, * * p<0.01 v s$. control. 

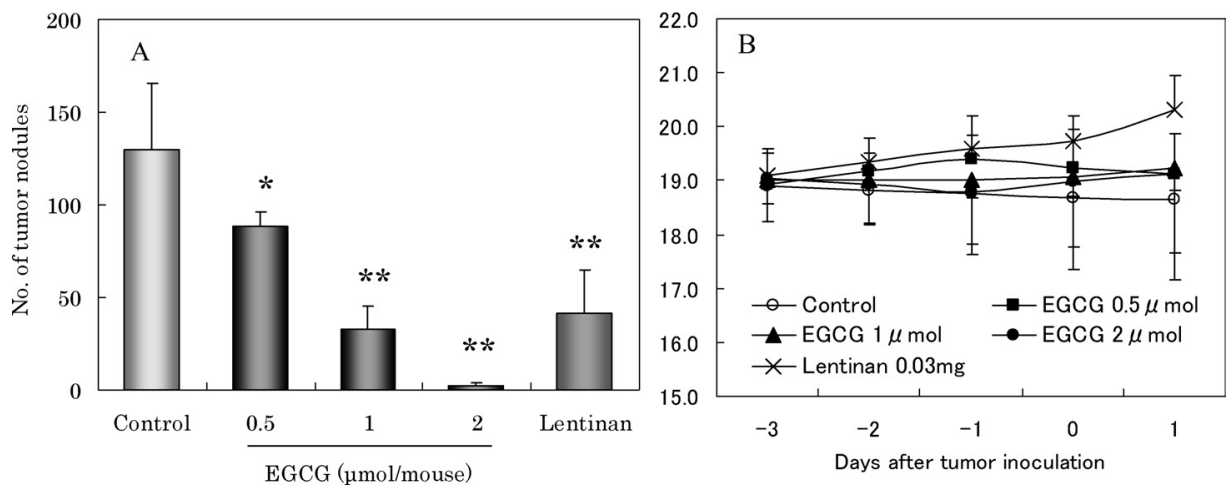

Fig. 2. Dose-Responsive Inhibition by EGCG of Lung Metastasis by Colon Carcinoma Cells in Mice

Colon 26-L5 cells were inoculated into mice through a tail vein. EGCG or Lentinan was administered intraperitoneally into mice for $5 \mathrm{~d}$ beginning at $3 \mathrm{~d}$ before tumor inoculation. Thirteen days after tumor inoculation, tumor nodules on the surfaces of the lungs were counted. The data are expressed as the mean \pm S.D. of 5 mice. ${ }^{*} p<0.05, * * p<0.01 v s$. control.

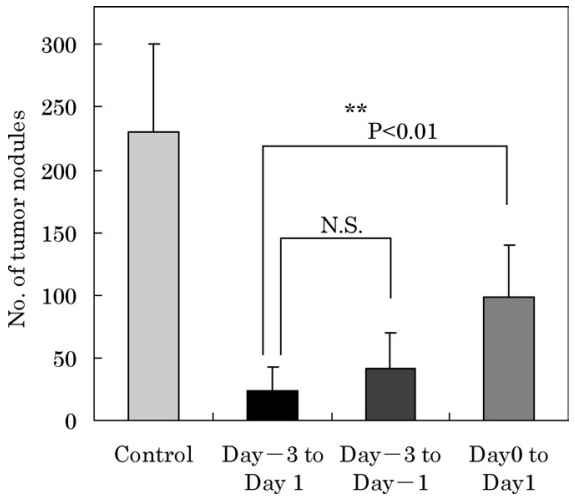

Fig. 3. Effects of the Administration of EGCG at Different Time Periods on the Lung Metastasis of Colon Carcinoma Cells in Mice

Mice were divided into four groups; they were administered $2 \mu \mathrm{mol}$ of EGCG at different time periods: for $5 \mathrm{~d}$ beginning at $3 \mathrm{~d}$ before tumor inoculation, for $3 \mathrm{~d}$ before the inoculation, and for $2 \mathrm{~d}$ after tumor inoculation. The control group was vehicle administered for $5 \mathrm{~d}$. Colon 26 -L 5 cells were inoculated into mice through a tail vein. Thirteen days later, tumor nodules on the surfaces of the lungs were counted. The data are expressed as the mean \pm S.D. of 5 mice. N.S.: not significant.

achieved 98\% suppression at a dose of $2 \mu \mathrm{mol}$; its effect at this dose was more potent than that of Lentinan, an anti-cancer agent, at a dose of $0.03 \mathrm{mg} /$ mouse. This dose of Lentinan was used as the optimal concentration for its antimetastatic activity in a mouse model, which we had determined using doses ranging from 0.01 to $0.1 \mathrm{mg} /$ mouse (data not shown). EGCG had little influence on the body weight of the mice (Fig. 2B).

We next examined the antimetastatic effect of EGCG at different time periods of its administration (Fig. 3). Pre-administration of EGCG before tumor inoculation was found to produce about $80 \%$ inhibition, which was equivalent to that produced by its complete administration through before to after the inoculation. On the other hand, post-administration of EGCG after tumor inoculation produced about 50\% inhibition; but it was statistically less effective than that produced by the complete administration.

Effect of Antioxidants on Tumor Cell Invasion Antioxidants were examined for their activity against Matrigel invasion of colon $26-\mathrm{L} 5$ cells in transwell chambers. $\mathrm{IC}_{50}$ values of less than $150 \mu \mathrm{m}$ were obtained for bilirubin, curcumin, genistein, quercetin, gallocatechin gallate, melatonin, resveratrol and EGCG, in order of the inhibitory activity
Table 1. Effects of Antioxidants on Tumor Cell Invasion, Growth and DPPH Radical Scavenging Activity

\begin{tabular}{lccc}
\hline \hline \multicolumn{1}{c}{ Antioxidants } & $\begin{array}{c}\text { Invasion } \\
\left(\mathrm{IC}_{50} \mu \mathrm{M}\right)\end{array}$ & $\begin{array}{c}\text { Growth } \\
\left(\mathrm{IC}_{50} \mu \mathrm{M}\right)\end{array}$ & $\begin{array}{c}\mathrm{DPPH} \text { radical } \\
\left(\mathrm{ED}_{50} \mu \mathrm{M}\right)\end{array}$ \\
\hline Ascorbic acid & $>150$ & $>50$ & 17.5 \\
Astaxanthin & $>150$ & $>50$ & $>50$ \\
Bilirubin & 14.0 & $>50$ & 12.1 \\
$\beta$-Carotene & $>150$ & $>50$ & $>50$ \\
Curcumin & 19.0 & 17.6 & 17.1 \\
L-Cystein & $>150$ & $>50$ & 32.2 \\
Epicatechin gallate & $>150$ & 34.0 & 4.5 \\
Epigallocatechin & $>150$ & $>50$ & $\mathrm{n} . \mathrm{d}$. \\
Epigallocatechin gallate & 148.2 & 41.8 & 2.9 \\
Folic acid & $>150$ & $>50.0$ & $>50$ \\
Genistein & 42.2 & 23.0 & 7.3 \\
Gallocatechin gallate & 97.4 & 32.5 & $\mathrm{n} . \mathrm{d}$. \\
Lycopene & $>150$ & $>50$ & $>50$ \\
Melatonin & 129.2 & $>50$ & $>50$ \\
Procyanidin B2 & $>150$ & $>50$ & 4.5 \\
Quercetin & 58.2 & 8.0 & $>50$ \\
$\alpha$-Tocopherol & $>150$ & $>50$ & 16.7 \\
Resveratrol & 131.4 & 15.8 & 54.2 \\
Rutin & $>150$ & $>50$ & 7.2 \\
Uric acid & $>150$ & $>50$ & $>50$ \\
\hline
\end{tabular}

n.d.: not determined.

(Table 1). Bilirubin and curcumin were 10 times and 7.5 times as potent as EGCG, respectively. No effect was observed in the case of $\alpha$-tocopherol, $\beta$-carotene and ascorbic acid at concentrations under $150 \mu \mathrm{M}$.

Effect of Antioxidants on Tumor Cell Proliferation Antioxidants were examined for their antiproliferative activity with respect to colon 26-L5 cells in vitro in a 48-h treatment. $\mathrm{IC}_{50}$ values of less than $50 \mu \mathrm{m}$ were obtained for quercetin, resveratrol, curcumin, genistein, gallocatechin gallate, epicatechin gallate and EGCG, in order of the inhibitory activity (Table 1). The activity of quercetin was 5.8 -fold higher than that of EGCG; $\alpha$-tocopherol, $\beta$-carotene and ascorbic acid had little effect at concentrations under $50 \mu \mathrm{M}$.

Free Radical Scavenging Activity of Antioxidants Antioxidants were examined for their free radical scavenging activity against $\mathrm{DPPH}$ radical. $\mathrm{ED}_{50}$ values under $50 \mu \mathrm{M}$ are indicated in Table 1. EGCG showed a 5.8-fold more potent scavenging activity than $\alpha$-tocopherol and a 6.1-fold more potent activity than ascorbic acid. Epicatechin gallate and 


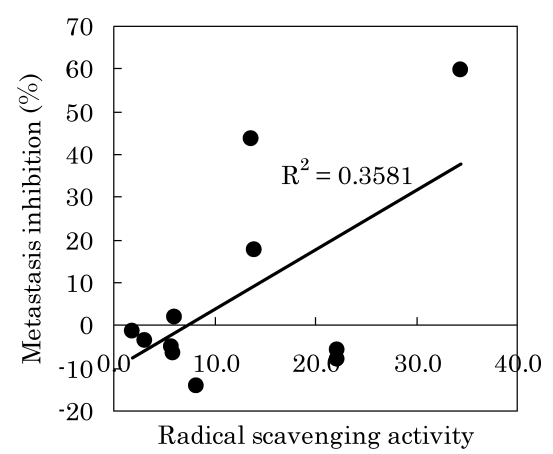

$\left[\left(1 / \mathrm{ED}_{50}\right) \times 100\right]$

Fig. 4. Relationship between the Free Radical Scavenging Activity of Antioxidants for DPPH Radical and Tumor Metastasis Inhibition

The stable radical DPPH was incubated with the test compounds, and the absorbance of the solution was then measured. The concentration needed to reduce the initial amount of $\mathrm{DPPH}$ by $50 \%\left(\mathrm{ED}_{50}\right)$ was determined. The data are expressed as $\left(1 / \mathrm{ED}_{50}\right) \times 100$.

procyanidin B2 exhibited an approximately two-thirds activity of EGCG. A slightly positive relationship was observed between the radical scavenging activities (indicated as $\left.\left(1 / \mathrm{ED}_{50}\right) \times 100\right)$ and the inhibition rates of tumor metastasis, but this relationship was not statistically significant (Fig. 4).

\section{DISCUSSION}

In the present study, we compared the antimetastatic activities of 20 antioxidants, including ascorbic acid, ${ }^{10)}$ astaxanthin, ${ }^{23)} \beta$-carotene, ${ }^{24)}$ curcumin, ${ }^{14)}$ EGCG, ${ }^{15)}$ genistein, ${ }^{16}$ quercetin, ${ }^{17)}$ rutin, ${ }^{18)}$ resveratrol ${ }^{19)}$ and $\alpha$-tocopherol, ${ }^{11)}$ which have been reported to be effective in inhibiting tumor metastasis in animal studies, finding that EGCG showed the most potent antimetastatic activity of the tested compounds. As described above, the antimetastatic activity of EGCG has previously been reported with respect to lung metastasis by murine B16 melanoma and Lewis lung carcinoma in animal models, ${ }^{15)}$ however, interestingly, the present results show that EGCG strongly prevented tumor metastasis formation without loss of body weight in the mice and more effectively than two highly notable compounds, curcumin and genistein. To the best of our knowledge, this is the first study showing the preventive action of EGCG on the formation of tumor metastasis. In a previous report on the antimetastatic activity of EGCG by Taniguchi et al., ${ }^{15)}$ the administration of EGCG was performed from the day before tumor inoculation until the end of the study period, showing the activity of EGCG as a therapeutic agent rather than a preventive one. In contrast, in the present study, EGCG was administered from $3 \mathrm{~d}$ before tumor inoculation to the next day of it, showing the activity of EGCG as a preventive agent. This preventive activity of EGCG can be further confirmed by the observation that just pre-administration of EGCG prior to tumor inoculation produced an equivalent inhibition to that produced by the above scheduled administration of EGCG. The present findings suggest that EGCG is a promising candidate as a preventive agent against tumor metastasis formation. This possibility might be further strengthened by two epidemiological investigations in which increasing the frequency of consumption of green tea extract, which contains EGCG as a main ingredient, was shown to be closely associated with lesser axillary lymph node metastasis in premenopausal patients with stage I and II breast cancer ${ }^{25)}$ and with longer survival in patients with epithelial ovarian cancer. ${ }^{26)}$

Structure-activity relationships of green tea catechins have been reported on some of their various biological activities. Makimura et al. showed that the 3-O-galloyl group was critical for the inhibition of collagenase activity; ${ }^{27}$ Oku et al. showed the data that catechins without the 3-O-galloyl group had much less inhibition against MT1-MMP activity, compared to ones with the group. ${ }^{28)}$ In addition, Isemura et al. reported that catechins with the pyrogallol-type B-ring structure could induce apoptosis of tumor cells effectively. ${ }^{29}$ In the present study, we have showed that EGCG inhibited tumor metastasis most effectively among EGCG, ECG, EGC, and GCG; and that ECG and EGC had no antimetastatic effect. These results suggest that the pyrogallol-type B-ring structure and the 3-O-galloyl group both are responsible for the antimetastatic activity of EGCG. We have also showed that antimetastatic activity of EGCG was much higher than that of GCG, suggesting that the cis-configuration of the pyrogallol-type B-ring structure against the 3-O-galloyl group could enhance the activity.

The antimetastatic mechanisms of EGCG have been suggested to include the inhibition of tumor cell invasion, ${ }^{30}$ growth $^{31)}$ and angiogenesis. ${ }^{32}$ We also observed the inhibitory activities of EGCG on the in vitro invasion and growth of tumor cells. However, these inhibitions are unlikely to be crucial mechanisms of the observed antimetastatic effect of EGCG, because of the following reasons: (1) only pre-administration of EGCG before tumor inoculation caused marked inhibition, (2) the concentrations active in vitro seem to be much higher than those in vivo, and (3) compounds showing no inhibitory activity against tumor metastasis in the present study, such as epicatechin gallate, curcumin and resveratrol, exhibited equivalent or even more potent inhibitory activities against tumor cell invasion and growth than EGCG. The inhibition of angiogenesis might also contribute in a limited way to the effect of EGCG, because tumor-induced angiogenesis would probably not occur aggressively at the times of the administration of EGCG in the present study. Tumor metastasis consists of a series of sequential steps: dissociation of tumor cells from the primary site, escape from the host immune system, translocation to distant organs through vessels, invasion into the surrounding tissues of those organs, and growth at the metastatic site. ${ }^{33)}$ Given these processes, possible key mechanisms of the antimetastatic activity of EGCG might be the inhibition of the interaction between tumor cells and endothelial cells or platelets, or the modulation of the host defense system. The inhibitory mechanisms of EGCG remain to be investigated.

Reactive oxygen species (ROS) have been suggested to increase the risk not only of carcinogenesis but also of tumor metastasis. ${ }^{34)}$ Nevertheless, we observed no statistically significant relationship between the radical scavenging activities of antioxidants and their inhibition rates of tumor metastasis. This observation implies that ROS may have had a marginal association with tumor metastasis formation under the experimental conditions of the present study. Thus, the antioxidative activity of EGCG may have little influence on its observed antimetastatic effect.

In conclusion, we have demonstrated that EGCG inhibited 
the lung metastasis of colon carcinoma in mice more potently than the other tested antioxidants, and that EGCG may be potentially beneficial for clinical applications as a preventive agent against tumor metastasis formation. Its antimetastatic mechanism seems to be independent of the inhibition of tumor cell invasion and growth, as well as of radical scavenging activity.

\section{REFERENCES}

1) Chambers A. F., MacDonald I. C., Schmidt E. E., Morris V. L., Groom A. C., Adv. Cancer Res., 79, 91-121 (2000).

2) Saiki I., Murata J., Matsuno K., Ogawa R., Nishi N., Tokura S., Azuma I., Jpn. J. Cancer Res., 81, 660-667 (1990).

3) Hidalgo M., Eckhardt S. G., J. Natl. Cancer Inst., 93, 178-193 (2001)

4) Ogasawara M., Matsubara T., Suzuki H., Biol. Pharm. Bull., 24, $917-$ 920 (2001).

5) Ishihara Y., Fujii T., Iijima H., Saito K., Matsunaga K., In Vivo, 12, 175-182 (1998)

6) Shirota K., Kato Y., Irimura T., Kondo H., Sugiyama Y., Biol. Pharm. Bull., 24, 316-319 (2001).

7) Amirkhosravi A., Mousa S. A., Amaya M., Blaydes S., Desai H., Meyer T., Francis J. L., Thromb. Haemost., 90, 549-554 (2003).

8) Yang C. S., Landau J. M., Huang M. T., Newmark H. L., Annu. Rev. Nutr., 21, 381-406 (2001).

9) Kanadaswami C., Lee L. T., Lee P. P., Hwang J. J., Ke F. C., Huang Y. T., Lee M. T., In Vivo, 19, 895-909 (2005).

10) Taper H. S., Jamison J. M., Gilloteaux J., Summers J. L., Calderon P. B., Life Sci., 75, 955-967 (2004).

11) Heukamp I., Kilian M., Gregor J. I., Neumann A., Jacobi C. A., Guski H., Schimke I., Walz M. K., Wenger F. A., Pancreatology, 5, 403-409 (2005).

12) Liu J. W., Nagao N., Kageyama K., Miwa N., Oncol. Res., 11, 479487 (1999).

13) Lawson K. A., Anderson K., Menchaca M., Atkinson J., Sun L., Knight V., Gilbert B. E., Conti C., Sanders B. G., Kline K., Mol. Can- cer Ther, 2, 437-444 (2003).

14) Ohashi Y., Tsuchiya Y., Koizumi K., Sakurai H., Saiki I., Oncology, 65, 250-258 (2003).

15) Taniguchi S., Fujiki H., Kobayashi H., Go H., Miyado K., Sadano H., Shimokawa R., Cancer Lett., 65, 51-54 (1992).

16) Li D., Yee J. A., McGuire M. H., Murphy P. A., Yan L., J. Nutr., 129, 1075-1078 (1999).

17) Mouria M., Gukovskaya A. S., Jung Y., Buechler P., Hines O. J., Reber H. A., Pandol S. J., Int. J. Cancer, 98, 761-769 (2002).

18) Menon L. G., Kuttan R., Kuttan G., Cancer Lett., 95, 221-225 (1995).

19) Kimura Y., Okuda H., J. Nutr., 131, 1844-1849 (2001).

20) Ohnishi Y., Sakamoto T., Fujii H., Kimura F., Murata J., Tazawa K., Fujimaki M., Sato Y., Kondo M., Une Y., Saiki I., Tumor Biol., 18, 113-122 (1997).

21) Saito K., Oku T., Ata N., Miyashiro H., Hattori M., Saiki I., Biol. Pharm. Bull., 20, 345-348 (1997).

22) Blois M. S., Nature (London), 181, 1199-1200 (1958).

23) Kurihara H., Koda H., Asami S., Kiso Y., Tanaka T., Life Sci., 70, 2509-2520 (2002).

24) Pradeep C. R., Kuttan G., Phytomedicine, 10, 159-164 (2003).

25) Nakachi K., Suemasu K., Suga K., Takeo T., Imai K., Higashi Y., Jpn. J. Cancer Res., 89, 254-261 (1998).

26) Binns C. W., Zhang M., Lee A. H., Xie C. X., Asia Pac. J. Clin. Nutr, 13, S116 (2004).

27) Makimura M., Hirasawa M., Kobayashi K., Indo J., Sakanaka S., Taguchi T., Otake S., J. Periodontol., 64, 630 -636 (1993).

28) Oku N., Matsukawa M., Yamakawa S., Asai T., Yahara S., Hashimoto F., Akizawa T., Biol. Pharm. Bull., 26, 1235-1238 (2003).

29) Isemura M., Saeki K., Kimura T., Hayakawa S., Minami T., Sazuka M., Biofactors, 13, 81-85 (2000).

30) Garbisa S., Sartor L., Biggin S., Salvato B., Benelli R., Albini A., Cancer, 91, 822-832 (2001).

31) Baliga M. S., Meleth S., Katiyar S. K., Clin. Cancer Res., 11, 1918 1927 (2005).

32) Fassina G., Vene R., Morini M., Minghelli S., Benelli R., Noonan D. M., Albini A., Clin. Cancer Res., 10, 4865-4873 (2004).

33) Poste G., Fidler I. J., Nature (London), 283, 139-146 (1980).

34) Brown N. S., Bicknell R., Breast Cancer Res., 3, 323-327 (2001). 